\title{
Transformation Pathways of Cocrystal Hydrates When Coformer Modulates Water Activity
}

\author{
ADIVARAHA JAYASANKAR, LILLY ROY, NAÍR RODRÍGUEZ-HORNEDO \\ Department of Pharmaceutical Sciences, University of Michigan, Ann Arbor, Michigan 48109-1065
}

Received 19 February 2010; accepted 21 April 2010

Published online 8 July 2010 in Wiley InterScience (www.interscience.wiley.com). DOI 10.1002/jps.22245

\begin{abstract}
An important attribute of cocrystals is that their properties can be tailored to meet required solubility and stability specifications. But before such practical uses can be realized, a better understanding of the factors that dictate cocrystal behavior is needed. This study attempts to explain the phase behavior of anhydrous/hydrated cocrystals when the coformer modulates both water activity and cocrystal solubility. Stability dependence on solution composition and water activity was studied for theophylline-citric acid (THP-CTA) anhydrous and hydrated cocrystals by both suspension and vapor equilibration methods. Eutectic points and associated water activities were measured by suspension equilibration methods to determine stability regions and phase diagrams. The critical water activity for the anhydrous-hydrate cocrystal was found to be 0.8 . It is shown that (a) both water and coformer activities determine phase stability, and (b) excipients that alter water activity can profoundly affect the hydrate/ anhydrous eutectic points and phase stability. Vapor phase stability studies demonstrate that cocrystals of highly water soluble coformers, such as citric acid, are predisposed to conversions due to moisture uptake and deliquescence of the coformer. The presence of such coformers as trace level impurities with cocrystal will alter hygroscopic behavior and stability. (c) 2010 WileyLiss, Inc. and the American Pharmacists Association J Pharm Sci 99:3977-3985, 2010
\end{abstract}

Keywords: hygroscopicity; transition concentration; eutectic point; phase diagram; stability

\section{INTRODUCTION}

The ability of cocrystals to increase drug thermodynamic activity, ${ }^{1-4}$ which may translate to improved bioavailability for poorly water soluble drugs, has deepened our awareness of solid phase stability during processing, storage, and dissolution. ${ }^{5-7}$ Cocrystals can exist as anhydrous and hydrated forms and convert to (a) hydrated or anhydrous components, in addition to (b) hydrated or anhydrous cocrystal. $^{4,8-12}$ For this reason, stability issues of cocrystals will be more challenging than those of its associated component crystals.

While the phase behavior of pharmaceutical hydrates is well established ${ }^{3,13-19}$ that of cocrystal hydrates is not. In the case of pharmaceutical hydrates, conversion to hydrate or anhydrous phases is mainly determined by the water activity, $a_{\mathrm{w}}$, of the surrounding medium (vapor or liquid phase). ${ }^{13,14,20-23}$

Adivaraha Jayasankar's present address is Global Formulation Sciences-Solid, Abbott Laboratories, AP31-4, 200 Abbott Park Road, Abbott Park, IL 60064.

Correspondence to: Naír Rodríguez-Hornedo (Telephone: 734763-0101; Fax: 734-615-6162; E-mail: nrh@umich.edu)

Journal of Pharmaceutical Sciences, Vol. 99, 3977-3985 (2010) (c) 2010 Wiley-Liss, Inc. and the American Pharmacists Association
Cocrystals however can follow different transformation pathways depending on the purity of the cocrystal phase and solution compositions. ${ }^{9,10,24-28}$ Thus, both water and solute activities are expected to play an important role on the stability of cocrystal phases.

Cocrystals not only alter aqueous solubility but also enable a wide range of solubility behaviors based on coformer selection, and in this way solve drug delivery problems. ${ }^{1,4,7}$ Hence, the advantages outweigh the perceived stability challenges. Drugs, such as carbamazepine (CBZ), theophylline, and caffeine, that exist as both anhydrous and hydrated crystals, form anhydrous cocrystals with several coformers. ${ }^{8,24,25,29,30}$ Some of these cocrystals have been shown to improve resistance to hydration although their component crystal phases are prone to hydration or to deliquescence (e.g., nicotinamide, maleic, malonic, citric acid (CTA), and other carboxylic acids). ${ }^{8,11,31}$ A few cocrystals are also reported to form both anhydrous and hydrated phases, ${ }^{12,24,32}$ yet the factors that control their formation and stability in solvents are not well known.

This report attempts to explain the transformation pathways of cocrystal anhydrous/hydrates when coformer modulates both $a_{\mathrm{w}}$ and cocrystal solubility. 
We first examine the stability dependence on solution composition and $a_{\mathrm{w}}$ for theophylline-citric acid (THP-CTA) anhydrous and hydrated cocrystals. CTA is very water soluble $\left(8.37 \mathrm{~m} \text { at } 25^{\circ} \mathrm{C}\right)^{33,34}$ and deliquescent, with saturation $a_{\mathrm{w}}$ of $0.78-0.79$ or deliquescence relative humidity (DRH) of $78-79 \%$ at $25^{\circ} \mathrm{C} .^{33-35}$ The THP-CTA cocrystal-solution phase diagram was generated from eutectic point measurements where two solid phases coexist in equilibrium with solution. Water activities at eutectic points were measured and related to the stability of the anhydrous/hydrated cocrystal phases in solutions of varying $a_{\mathrm{w}}$ and in vapor phase in a range of $\mathrm{RH}$.

Additives used in pharmaceutical formulations can also lower $a_{\mathrm{w}}$ and to this effect the cocrystal hydrate/ anhydrous stability and associated eutectic point were assessed in solutions of fructose. Since CTA is deliquescent, the $\mathrm{RH}$-dependent transformation pathways of binary mixtures of cocrystal components were investigated, and are explained by the solutionphase behavior of cocrystal and its components. Finally, the stability of carbamazepine-4 amino benzoic acid (CBZ-4ABA) hydrate/anhydrous as a function of $a_{\mathrm{w}}$ was measured. As with THP-CTA, this cocrystal is more soluble than the drug and converts to CBZ dihydrate when suspended in water. ${ }^{4}$ However, the coformer in this case, 4ABA, has lowaqueous solubility ${ }^{36}$ and is less effective in lowering $a_{\mathrm{w}}$. These findings have significant implications for determining the factors that control cocrystal stability and conversions. Furthermore, since the phase at equilibrium is determined by solution composition and ambient water activity, the purity of cocrystal phase will greatly affect stability.

\section{EXPERIMENTAL}

\section{Materials}

Anhydrous THP form (II), anhydrous CBZ form (III), and 4-aminobenzoic acid (4ABA) form $(\alpha)$ used in the studies were purchased from Sigma-Aldrich, St. Louis, MO. Anhydrous CTA was purchased from Fisher Scientific, NJ. All chemicals were characterized by X-ray powder diffraction (XRPD) and Raman spectroscopy prior to use. Ethanol and 2-acetonitrile, purchased from Acros, NJ, were dried using molecular sieves prior to use.

\section{Cocrystal Synthesis}

Cocrystals were prepared by the reaction crystallization method at room temperature by adding drug to nonstoichiometric solutions of coformer. ${ }^{27,37}$ Anhydrous THP-CTA (1:1) and CBZ-4ABA (2:1) cocrystals were prepared in ethanol. THP_CTA hydrate (1:1:1) and CBZ-4ABA hydrate (2:1:1) cocrystals were prepared in water. Solid phases were isolated and characterized by XRPD and Raman spectroscopy.

\section{Suspension Equilibration Studies}

THP_CTA hydrate stability dependence on coformer solution concentration was examined by suspending excess cocrystal hydrate in aqueous solutions with varying CTA concentrations. The suspensions were magnetically stirred in a water bath at $25.0 \pm 0.5^{\circ} \mathrm{C}$ for $24-48 \mathrm{~h}$ prior to adding anhydrous cocrystal. The suspensions were stirred for 2-3 weeks to reach equilibrium. Solution concentrations of CTA at equilibrium were measured by HPLC. Equilibrium was considered to be achieved when the solution concentrations did not change by more than $2-3 \%$. Solid phases were characterized by Raman spectroscopy and XRPD. The water activities of the equilibrated suspensions were determined by measuring the RH above the suspensions using a Hydroclip $\mathrm{RH}$ probe from Rotronics (Huntington, $\mathrm{NY})$. Probe accuracy is $\pm 1.5 \% \mathrm{RH} / \pm 0.2^{\circ} \mathrm{C}$.

THP-CTA hydrate stability dependence on water activity was studied by suspending excess THP-CTA hydrate in aqueous fructose-CTA solutions with varying fructose concentration but constant CTA concentration $(6.05 \mathrm{~m})$ at $25.0 \pm 0.5^{\circ} \mathrm{C}$. The suspensions were allowed to reach equilibrium as described above. Solid phase stability in suspensions was periodically monitored using Raman spectroscopy and XRPD. The water activity of the suspensions was determined by measuring the $\mathrm{RH}$ as described above.

CBZ-4ABA hydrate stability dependence on water activity was studied in water/acetonitrile solutions since the anhydrous cocrystal was stable in acetonitrile. Solutions of water and acetronitrile ranging from mole fraction of water $\left(x_{\mathrm{w}}\right) 0$ to 0.054 with water activities between 0 and 0.41 were prepared based on literature values. ${ }^{38}$ The suspensions were magnetically stirred for $48 \mathrm{~h}$ at $25.0 \pm 0.5^{\circ} \mathrm{C}$ prior to adding anhydrous cocrystal. The suspensions were then stirred for an additional 2 weeks. Solid phases were characterized by Raman spectroscopy and XRPD to monitor phase transformations.

\section{Eutectic Concentration Measurement}

Three eutectic points were examined for the THPCTA cocrystal in water where the following phases coexist in equilibrium with solution: eutectic $\left(E_{1}\right)$ THP hydrate and cocrystal hydrate, eutectic $\left(E_{2}\right)$ cocrystal hydrate and anhydrous cocrystal, and eutectic $\left(E_{3}\right)$ anhydrous cocrystal and CTA monohydrate. Eutectic concentrations of THP and CTA were measured by HPLC after equilibrating the two solid phases (as indicated for $E_{1}, E_{2}$, and $E_{3}$ ) with aqueous solutions at $25.0 \pm 0.5^{\circ} \mathrm{C}$. Equilibrium was considered to be achieved when solution concentrations reached a constant value while two solid phases coexist. The 
solid phases in suspension were initially characterized by Raman spectroscopy. The suspensions were filtered and the solid phases were characterized by XRPD. Water activities corresponding to the eutectic concentrations were determined by measuring the $\mathrm{RH}$ above the equilibrated suspensions as described above.

\section{RH-Dependent Solid Phase Stability}

\section{Cocrystal}

The moisture uptake and stability of THP-CTA and CBZ-4ABA cocrystals (anhydrous and hydrated) was examined at several $\mathrm{RH}$ values. THP-CTA (1:1) and THP-CTA hydrate $(1: 1: 1)$ were studied at $85 \%$ and $98 \% \mathrm{RH}$ at $25.0 \pm 0.5^{\circ} \mathrm{C}$. CBZ-4ABA (2:1) and CBZ4ABA hydrate (2:1:1) were studied at $0 \%, 13 \%, 43 \%$, and $89 \% \mathrm{RH}$ at room temperature $\left(24 \pm 1^{\circ} \mathrm{C}\right)$. Desired $\mathrm{RH}$ conditions were achieved in sealed desiccators containing the appropriate saturated salt solution: $\mathrm{P}_{2} \mathrm{O}_{5}(0 \%), \mathrm{LiCl} \cdot \mathrm{H}_{2} \mathrm{O}(13 \%), \mathrm{K}_{2} \mathrm{CO}_{3} \cdot 2 \mathrm{H}_{2} \mathrm{O}(43 \%), \mathrm{NaCl}$ (75\%), $\mathrm{KCl}(85 \%), \mathrm{BaCl}_{2} \cdot 2 \mathrm{H}_{2} \mathrm{O}(89 \%)$, and $\mathrm{K}_{2} \mathrm{SO}_{4}$ $(98 \%){ }^{39,40}$ Samples $(30-60 \mathrm{mg}, 45-63 \mu \mathrm{m})$ were analyzed by XRPD prior to and after exposure to the different $\mathrm{RH}$ conditions.

\section{Mixtures of Cocrystal Components}

The role of moisture uptake on cocrystal formation in equimolar mixtures $(30-60 \mathrm{mg}, 45-63 \mu \mathrm{m})$ of anhydrous THP and anhydrous CTA was studied in desiccators equilibrated to $75 \%, 85 \%$, and $98 \% \mathrm{RH}$ at $25.0 \pm 0.5^{\circ} \mathrm{C}$ using the procedures described in an earlier study. ${ }^{41}$ Mixtures were analyzed by XRPD prior to introduction into desiccators. Phase transformations in closed desiccators were monitored by Raman spectroscopy. Spectra were collected frequently over random areas of the sample for several days. HoloReact software, from Kaiser Optical Systems (Ann Arbor, MI), was used for multivariate curve resolution to plot the change in spectral features correlating to reactants and cocrystal. The analysis region for the THP-CTA systems was 1600 $1750 \mathrm{~cm}^{-1}$. Change in spectral features over time was used to monitor cocrystal formation. Samples were promptly analyzed by XRPD once removed from the desiccator.

\section{Raman Spectroscopy}

Raman spectra of solid phases were collected with an RXN1 Raman spectrometer equipped with a $785 \mathrm{~nm}$ laser from Kaiser Optical System, Inc. Solid phase transformation in samples was monitored using an immersion or noncontact fiber optic probe. The immersion probe was used to collect the spectra of solid phases in aqueous suspensions and the noncontact probe was used to monitor transformations in solid mixtures during storage. Acquisition conditions were optimized so that the spectra collected had maximum intensity $(30-40 \mathrm{k})$. The spectra were collected between 100 and $1800 \mathrm{~cm}^{-1}$ with a resolution of $4 \mathrm{~cm}^{-1}$.

\section{X-Ray Powder Diffraction}

$\mathrm{XRPD}$ was used to identify solid phases recovered from the suspension equilibration studies, eutectic measurements, and the vapor phase equilibration studies. XRPD patterns of solid phases were recorded with a Rigaku Miniflex X-ray powder diffractometer (Danvers, MA) using $\mathrm{Cu} \mathrm{K} \alpha$ radiation $(\lambda=1.54 \AA)$, a tube voltage of $30 \mathrm{kV}$, and a tube current of $15 \mathrm{~mA}$. The intensities were measured at $2 \theta$ values from $2^{\circ}$ to $40^{\circ}$ at a continuous scan rate of $2.5^{\circ} \mathrm{min}^{-1}$. Results were compared to diffraction patterns reported in literature or calculated from crystal structures reported in the Cambridge Structural Database (CSD).

\section{High-Performance Liquid Chromatography (HPLC)}

Solution concentrations of THP and CTA were analyzed by Waters HPLC (Milford, MA) equipped with a UV/vis spectrometer detector. A C18 Atlantis column ( $5 \mu \mathrm{m}, 4.6 \mathrm{~mm} \times 250 \mathrm{~mm}$; Waters) at ambient temperature was used to separate THP and CTA. A gradient method, starting with $40 \%$ methanol and $0.1 \%$ trifluoroacetic acid in water and increasing methanol to $95 \%$, was used with a flow rate of $1 \mathrm{~mL} \mathrm{~min}^{-1}$. Sample injection volume was $20 \mu \mathrm{L}$. Absorbance of the THP and CTA was monitored between 210 and $300 \mathrm{~nm}$. Empower software from Waters was used to collect and process the data. All concentrations are reported in molality (moles solute/ kilogram solvent) unless otherwise indicated.

\section{RESULTS}

\section{Effect of Coformer Concentration on THP-CTA Cocrystal Hydrate Stability}

Cocrystal hydrate stability was initially investigated by suspension equilibration in aqueous solutions of CTA of several concentrations. Cocrystal hydrate stability and conversions were found to be dependent on coformer concentration as summarized in Figure 1, and as indicated by XRPD patterns of solid phases (Supporting Information). THP-CTA hydrate was found to be stable at CTA concentrations between 2.39 and $6.80 \mathrm{~m}$. Cocrystal hydrate transformed to THP hydrate at CTA concentrations below $1.62 \mathrm{~m}$, and to THP-CTA cocrystal anhydrous above $7.31 \mathrm{~m}$. Thus, coformer solution concentration not only determines conversions between cocrystal and components but between anhydrous and hydrated cocrystal phases. 


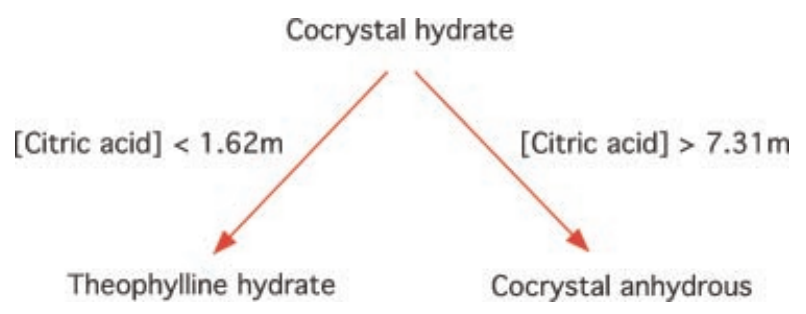

Figure 1. Dependence of theophylline-citric acid cocrystal hydrate stability on the concentration of citric acid in solution.

\section{Eutectic Points and Phase Diagram of THP-CTA Cocrystals}

Cocrystal eutectic points or transition concentrations, where two solid phases coexist in equilibrium with solution, have been shown to be key indicators of cocrystal stability. ${ }^{1,37,42}$ Results presented above for THP-CTA cocrystals suggest that this system exhibits three eutectic points $\left(E_{1}, E_{2}\right.$, and $\left.E_{3}\right)$. Solution concentrations of cocrystal components at the eutectic points and the associated solid phase equilibria were measured and are presented in Table 1 . The solid phases in equilibrium with solution at $E_{1}, E_{2}$, and $E_{3}$ were found to be drug hydrate/cocrystal hydrate, cocrystal hydrate/anhydrous cocrystal, and anhydrous cocrystal/coformer hydrate, respectively.

Table 1 shows that (THP) $)_{\text {eu }}$ decreases as $(\text { CTA })_{\text {eu }}$ increases from eutectic points $E_{1}$ to $E_{2}$. In the region above $E_{1}$ and below $E_{2}$, cocrystal hydrate is the thermodynamically stable phase. Therefore, its solubility, represented by the THP concentrations, decreases with increasing coformer concentration. Above $E_{2}$ and below $E_{3}$, anhydrous cocrystal is the thermodynamically stable phase. Coformer solution composition is thus controlling cocrystal solubility as well as its hydrated state.

A phase solubility diagram (PSD) generated from eutectic point measurements is shown in Figure 2. This plot indicates stability domains of solid phases and associated solution concentrations. The figure was constructed from experimentally measured eutectic concentrations and cocrystal solubility dependence on coformer concentration as previously reported for other systems. ${ }^{1,24,37}$ In addition to transformation between cocrystal hydrate/anhydrous, there are also regions where cocrystal transforms to either drug hydrate or coformer hydrate.
THP hydrate solubility is indicated by the line a- $E_{1}$ and CTA solubility by b- $E_{3}$. Solubility of both components is observed to increase compared to pure solvent.

\section{Solution Water Activity and THP-CTA Cocrystal Stability}

Conversion of hydrated to anhydrous cocrystal at coformer concentrations above $E_{2}$ is explained by the effect of coformer concentration on water activity, a factor well known to determine the stability of pharmaceutical hydrates. ${ }^{13,14,20-23}$ Water activity of solutions at eutectic points $E_{1}$ and $E_{2}$ and of solution compositions in between $E_{1}$ and $E_{3}$ were measured and are plotted in Figure 3. Water activities of THP/ CTA solutions are slightly lower $(\sim 3 \%)$ than those reported in the literature for aqueous solutions of CTA. ${ }^{34,43-45}$ This may be due to the presence of THP as well as differences in the methods used to measure water activity.

XRPD patterns of solid phases at equilibrium (Supporting Information) corresponding to solution concentrations and water activities presented in Figure 3 demonstrate that the critical water activity where hydrated and anhydrous cocrystals are in equilibrium is 0.8 . Above this $a_{\mathrm{w}}$ anhydrous cocrystal converted to its hydrated form and below this value the reverse conversion was observed.

Besides coformer, other excipients such as sugars used in pharmaceutical formulations can also lower the water activity of solutions. Fructose is reported to lower the water activity of aqueous solutions to 0.64 at saturation. ${ }^{35,46}$ This value is below the critical $a_{\mathrm{w}}$ for the anhydrous/hydrate THP-CTA cocrystal. Thus, varying fructose concentrations in cocrystal suspensions can lead to conversions between anhydrous and hydrated forms of the cocrystal.

Figure 3 shows the effect of fructose on $a_{\mathrm{w}}$ and cocrystal hydrate stability in aqueous THP/CTA solutions. Since cocrystal hydrate transforms to crystalline drug hydrate in water without CTA, the effect of fructose on cocrystal hydrate stability was investigated in coformer solutions where cocrystal hydrate is stable. XRPD patterns of solid phases at equilibrium (Supporting Information) demonstrate that as $a_{\mathrm{w}}$ is lowered from 0.84 to 0.79 , by addition of fructose to $6.05 \mathrm{~m}$ CTA solutions, cocrystal hydrate converts to its anhydrous form. This also corresponds

Table 1. Eutectic Concentrations $\left(C_{\text {eu }}\right)$ and Solid Phases at Equilibrium With THP/CTA Aqueous Solutions at $25^{\circ} \mathrm{C}$

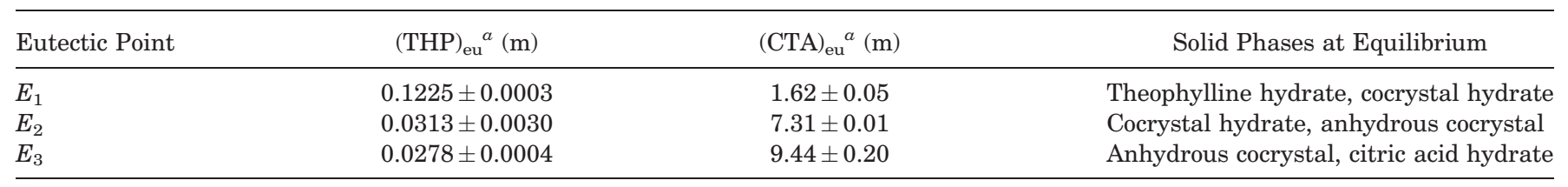

${ }^{a}$ Concentrations are mean \pm standard deviation. 


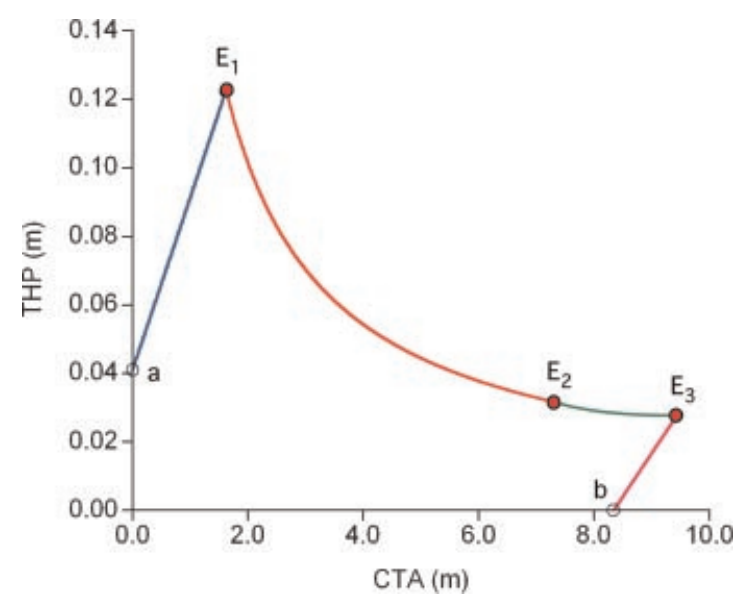

Figure 2. Phase solubility diagram of theophylline-citric acid cocrystals showing the stability domains and solubility dependence on coformer concentrations for the different solid phases. $E_{1}, E_{2}$, and $E_{3}$ correspond to eutectic points. "a" and "b" represent the aqueous solubilities of theophylline hydrate and citric acid hydrate as reported in the literature. $^{33,34,50}$ Lines through a, $E_{1}, E_{2}, E_{3}$, and b are drawn based on the behavior of the cocrystal solubility dependence on coformer for other cocrystal systems. The stability domains of crystalline phases are: a- $E_{1}$ : theophylline hydrate, $E_{1} E_{2}$ : cocrystal hydrate, $E_{2} E_{3}$ : anhydrous cocrystal and $\mathrm{bc}_{3}$ : citric acid hydrate.

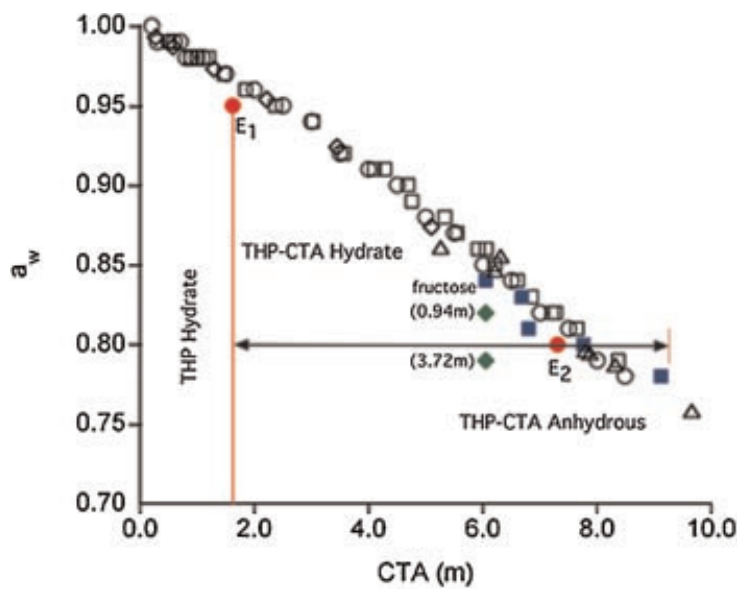

Figure 3. Effect of water activity on THP-CTA cocrystal hydrate/anhydrous stability. Line indicates the critical water activity for the anhydrous/hydrate THP-CTA cocrystal equilibrium. Water activity as a function of citric acid concentration in (a) citric acid aqueous solutions $\left(\square^{43} \bigcirc^{34}\right.$ $\diamond^{44} \Delta^{45,51}$ ), (b) theophylline and citric acid aqueous solutions at eutectic points: $\mathrm{E}_{1}$ in equilibrium with THP hydrate/cocrystal hydrate, and $\mathrm{E}_{2}$ in equilibrium with cocrystal hydrate/anhydrous (O), and in equilibrium with cocrystal hydrate or anhydrous ( $\square$ ), and (c) in solutions of fructose at constant citric acid concentration in equilibrium with cocrystal hydrate or anhydrous $(\bullet)$. to a decrease in eutectic concentrations at $\mathrm{E}_{2}$ as observed for $(\mathrm{CTA})_{\text {eu }}$, from 7.31 to $6.05 \mathrm{~m}$. Thus, the main factor controlling the cocrystal hydrate to anhydrous stability is $a_{\mathrm{w}}$, which in this case is modulated by both coformer and sugar.

\section{RH-Dependent Stability of THP-CTA Cocrystal}

The stability of THP-CTA cocrystals was evaluated at $85 \%$ and $98 \% \mathrm{RH}$ for 5 months. Both anhydrous and hydrated cocrystals were found to be stable at $85 \%$ $\mathrm{RH}$ whereas at $98 \% \mathrm{RH}$ both cocrystals converted to THP hydrate. The critical $a_{\mathrm{w}}$ of 0.8 determined by suspension equilibration suggests that conversion by vapor phase equilibration may be kinetically controlled. Similar behavior by vapor and suspension equilibration has been shown for THP hydrate and anhydrous. ${ }^{13,17,22,47}$

The cocrystal hydrate has been reported to be stable at $98 \% \mathrm{RH}$, however, the length of storage was only 1 week. ${ }^{12}$ The reasons for this difference in behavior at $98 \% \mathrm{RH}$ may be explained by longer storage times required for conversion and/or by the existence of coformer as an impurity in cocrystal samples. We observed deliquescence at $98 \% \mathrm{RH}$ for both cocrystal phases with significant water uptake at 5 months $(>70 \%, \mathrm{w} / \mathrm{w})$. At $85 \% \mathrm{RH}$, the water uptake was in the range of $4-6 \%$. DSC analysis of several cocrystal samples in our studies revealed coformer impurity between $0.5 \%$ and $5 \%(\mathrm{w} / \mathrm{w})$. CTA is deliquescent and trace levels in cocrystal samples stored at or above its deliquescent $\mathrm{RH}$ could lead to conversions via solution phase. It is also noted that caffeine-CTA cocrystal has been reported to deliquesce at $98 \%$ and convert to caffeine hydrate in 1 week. ${ }^{12}$

\section{Moisture Sorption of Cocrystal Components THP/CTA and RH-Dependent Transformations}

Results from the above studies show that solution composition and corresponding coformer/water activities determine transformation pathways of cocrystals to hydrated, anhydrous cocrystal phases or to hydrated phases of components. Since CTA is a water soluble, deliquescent coformer, it is possible for moisture sorption of mixtures to cause phase changes. $\mathrm{RH}$-dependent behavior of equimolar mixtures of THP/CTA summarized in Figure 4 was studied by $\mathrm{XRPD}$ and Raman spectroscopy (Supporting Information). Formation of cocrystal was found to occur at all RH conditions studied, but the pathways were dependent on $\mathrm{RH}$. The critical $a_{\mathrm{w}}$ for THP hydrate has been reported as 0.25 at $25^{\circ} \mathrm{C}{ }^{13}$ and 0.6 at $22^{\circ} \mathrm{C}^{22}$ Anhydrous cocrystal was formed initially at all $\mathrm{RH}$ values, and converted to hydrated cocrystal and THP hydrate as $\mathrm{RH}$ values increased above the CTA DRH of $78-79 \% .^{33-35}$ Anhydrous cocrystal was formed 


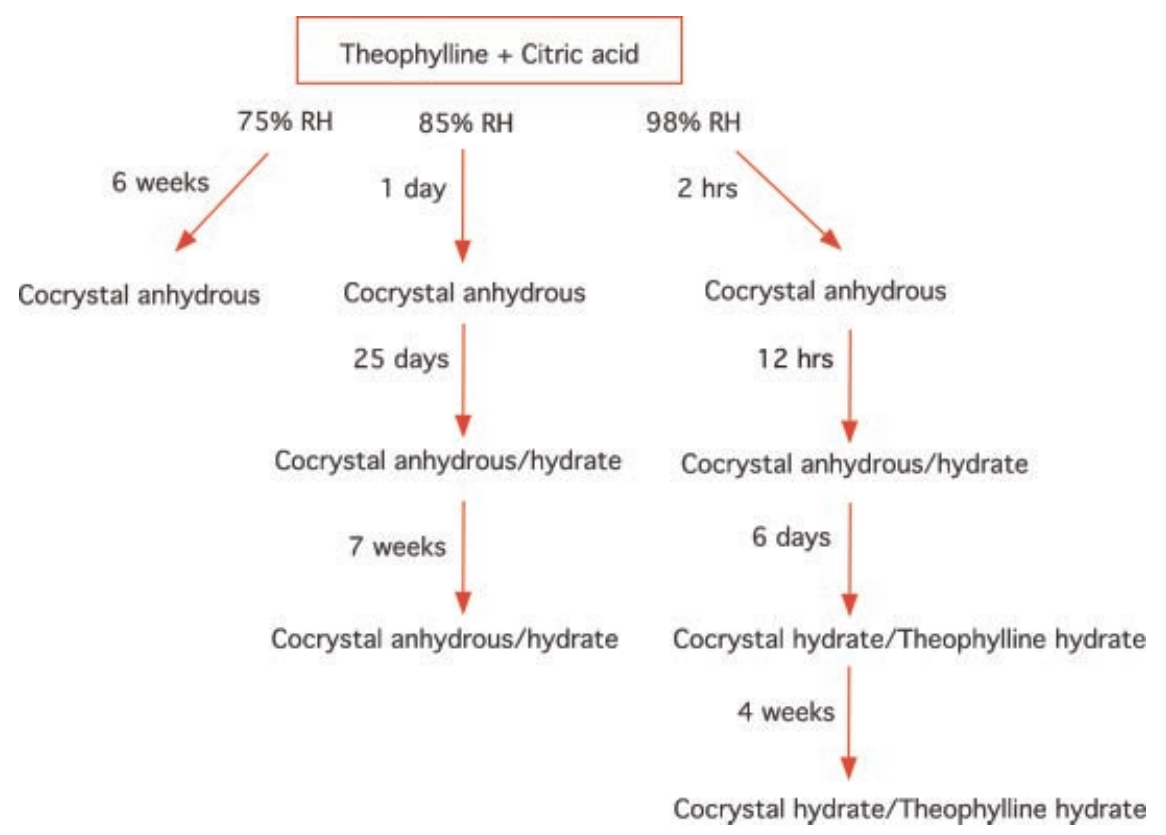

Figure 4. Transformation pathways of equimolar mixtures of anhydrous THP and anhydrous CTA exposed to different RH. Total storage times are indicated.

within weeks at $75 \% \mathrm{RH}$ and within a few hours at 98\% RH.

Initial formation of anhydrous cocrystal and subsequent conversion to other phases is explained by the phase equilibrium dependence on solution composition and $a_{\mathrm{w}}$. As shown by eutectic points and PSD (Fig. 3 and Tab. 1), conversion of a mixture of cocrystal components to anhydrous cocrystal $\rightarrow$ hyhydrated cocrystal $\rightarrow$ THP hydrate is associated with high initial CTA concentrations (between $\mathrm{E}_{3}$ and $\mathrm{E}_{2}$ ) and further dilution by moisture uptake leading to conditions where hydrated cocrystal (between $E_{2}$ and $\left.E_{1}\right)$ or THP hydrate ( $E_{1}$ and below) are in equilibrium.

Cocrystal component concentrations and activities are higher in the earlier stages of moisture uptake (small volumes of sorbed moisture) and decrease as the moisture sorption and deliquescence proceed (larger volumes of moisture will be sorbed at higher $\mathrm{RH})$. Thus the interplay of water and component activities in the deliquesced liquid determines the conversions between component and cocrystal phases.

\section{Water Activity and Stability of CBZ-4ABA Cocrystals}

Previous studies from our laboratory have shown that the hydrated CBZ-4ABA cocrystal is unstable in pure water and transforms to carbamazepine dihydrate. The eutectic points for the equilibrium between CBZ4ABA hydrate and CBZ dihydrate as a function of coformer concentration and $\mathrm{pH}$ were also reported. ${ }^{4}$ The hydrated cocrystal was found to be stable in water saturated with $4 \mathrm{ABA}$. Therefore in the present study, the influence of $a_{\mathrm{w}}$ on the stability of $2: 1 \mathrm{CBZ}-$
4ABA anhydrous/hydrated cocrystals was measured in water/acetonitrile solutions. XRPD patterns of solid phases at equilibrium (Supporting Information) demonstrate that anhydrous cocrystal is stable at $a_{\mathrm{w}} \leq 0.26$ and cocrystal hydrate is stable at $a_{\mathrm{w}} \geq 0.30$. Thus, the critical water activity above which the cocrystal hydrate is thermodynamically stable is $0.26-0.30$.

Vapor phase equilibration studies showed that both anhydrous and hydrated cocrystals resist conversion under conditions far from the equilibrium $a_{\mathrm{w}}$ measured by suspension. Anhydrous cocrystal stored at $43 \% \mathrm{RH}$ (5 months) and $89 \% \mathrm{RH}$ (1 month), and hydrated cocrystal stored at $0 \% \mathrm{RH}$ (1 month) and $13 \%$ RH (5 months) did not show evidence of conversion by XRPD analysis.

\section{DISCUSSION}

Cocrystals can exist as anhydrous and hydrated forms and convert to (a) hydrated or anhydrous components in addition to (b) hydrated or anhydrous cocrystal. ${ }^{4,8-12}$ Results from this study show that cocrystal hydrate/anhydrous stability is dependent on coformer concentration indicating that both coformer and water activities determine phase stability. Depending on coformer concentration, cocrystal hydrate can transform to crystalline drug or anhydrous cocrystal. Conversions between anhydrous and hydrated cocrystals occur when coformer and/or excipients modulate water activity of solutions as demonstrated for THP-CTA cocrystal hydrate/anhy- 
drous. CBZ-4ABA did not exhibit such solutionmediated conversion since $4 \mathrm{ABA}$ has low-aqueous solubility with little effect on water activity. The $a_{\mathrm{w}}$ of saturated aqueous $4 \mathrm{ABA}\left(a_{\mathrm{w}}=0.98\right)$ is much higher than the critical $a_{\mathrm{w}}$ of CBZ-4ABA hydrate $\left(a_{\mathrm{w}}=0.26\right.$ $0.30)$.

Cocrystals of highly water soluble coformers, such as THP-CTA, are predisposed to conversions due to moisture uptake and deliquescence of the coformer. This behavior may explain the RH-dependent stability reported for cocrystals of THP and caffeine with a series of carboxylic acids (malonic, maleic, glutaric, Dmalic, malic, D-tartaric, and tartaric). ${ }^{8,11,25}$ Resistance to conversion to hydrated drug at $98 \% \mathrm{RH}$ appears to be correlated with the solubility and hygroscopicity of the coformers. The coformer with the lowest solubility and hygroscopicity, oxalic acid $(S=1.3 \mathrm{~m}, \mathrm{DRH}=98 \%),{ }^{48}$ exhibited the best cocrystal stability at $98 \% \mathrm{RH}$. The most soluble and hygroscopic coformer, D-malic acid $(S=19.5 \mathrm{~m}$, $\mathrm{DRH}=56 \%),{ }^{48}$ was the only cocrystal unstable at $75 \%$ RH. Cocrystals with malonic $(S=15.3 \mathrm{~m}$, $\mathrm{DRH}=73 \%),{ }^{48}$ glutaric $(S=10.7 \mathrm{~m}, \mathrm{DRH}=88 \%),{ }^{48}$ and D-malic converted to the hydrated drug at $98 \%$ $\mathrm{RH}$. This behavior is paralleled by cocrystals composed of the racemic versus chiral coformers. ${ }^{25}$ The latter have higher solubility and hygroscopicity than the racemic forms. As with our results for THP-CTA cocrystal stability dependence on $\mathrm{RH}$, it is not clear whether the observed instability reflects that of the cocrystal or is due to coformer impurities. Trace levels of coformer undetected by XRPD can significantly affect the stability of cocrystals when the coformer $\mathrm{DRH}$ is at or below ambient $\mathrm{RH}$.

Eutectic points are key parameters to identify cocrystal stability domains. ${ }^{1,37,42}$ From the eutectic concentrations, PSD showing the reactant solution concentrations at equilibrium with the different solid phases as well as the cocrystal stability domains can be plotted as shown in Figure $3 .^{24,37}$ Alternatively, the stability domains can also be represented on a triangular phase diagram (TPD) that shows the total composition of the system, including the solid and liquid phases. ${ }^{10,20,24,26}$ Figure 5 shows a schematic TPD where the coformer modulates the water activity similar to THP/CTA/water system. Given the large differences in drug and coformer solubilities in the THP/CTA/water system, a hypothetical ternary system is presented for visual clarity to qualitatively show the transformation pathways by moisture sorption or when solid phases are in contact with solutions.

Transformation pathways and crystallization outcomes can be predicted from the phase diagram as represented by the pathway " $R$ " for deliquescence of a component in a solid mixture. During deliquescence, supersaturation with respect to cocrystal is generated

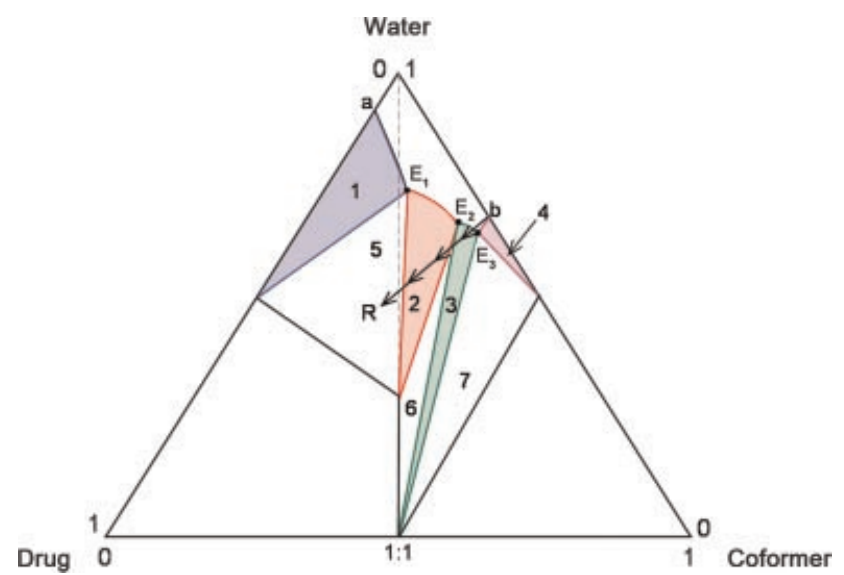

Figure 5. Triangular phase diagram showing the stability domains for anhydrous and hydrated cocrystals with coformers that modulate the water activity. Points "a" and "b" correspond to API hydrate and coformer hydrate aqueous solubility. $E_{1}, E_{2}$, and $E_{3}$ represent eutectic points. Curves a $E_{1}, E_{1} E_{2}, E_{2} E_{3}$, and $E_{3}$ b represent the solubilities of crystalline drug hydrate, cocrystal hydrate, anhydrous cocrystal, and hydrated coformer, respectively. Stability regions for the crystalline phases are: 1, crystalline drug hydrate; 2 , cocrystal hydrate; 3 , anhydrous cocrystal; 4, coformer hydrate; 5 , crystalline drug hydrate/cocrystal hydrate; 6, anhydrous/hydrated cocrystals; 7, anhydrous cocrystal/hydrated coformer. Pathway $\mathrm{R}$ represents the transformation occurring by reaction crystallization or deliquescence of solid mixtures during storage.

by nonequivalent cocrystal component concentrations in the deliquesced solution. As deliquescence begins, the sorbed water is saturated with coformer (generally the more soluble component). ${ }^{41,49}$ Under these conditions, the anhydrous cocrystal is the least soluble and most stable phase. The dissolution of drug in this solution therefore generates supersaturation with respect to anhydrous cocrystal. Further transformation of anhydrous cocrystal to cocrystal hydrate will occur when supersaturation with respect to cocrystal hydrate is generated. This is achieved when the coformer concentration falls below that corresponding to the eutectic point $E_{2}$.

The concentration of the coformer in a deliquesced solution depends on the RH. At the DRH, the deliquesced solution remains saturated with the coformer. However, at higher $\mathrm{RH}(\mathrm{RH}>\mathrm{DRH})$, increased levels of water uptake lead to coformer dilution in the deliquesced solution thereby affecting cocrystal stability. Coformer dilution at high $\mathrm{RH}$ explains the observed transformation pathway in THP/CTA mixtures at $85 \%$ and $98 \% \mathrm{RH}$.

A previous study from our laboratory showed that cocrystals of CBZ-nicotinamide, caffeine and THP with oxalic, mandelic, malonic, and glutaric acids form, in mixtures of coformer and drug, as a result of coformer deliquescence. ${ }^{41}$ Although these cocrystals 
convert to drug hydrate in pure water, their formation in mixtures of components was explained by dissolution of reactants in the deliquesced liquid phase leading to nonstoichiometric solution compositions and supersaturation with respect to cocrystal.

The critical $a_{\mathrm{w}}$ of CBZ-4ABA and THP-CTA hydrates is different from those of the drug hydrates. The critical $a_{\mathrm{w}}$ for CBZ and THP hydrates is reported as $0.64-0.65^{20,21}$ and $0.25-0.6,{ }^{13,22}$ respectively. The critical $a_{\mathrm{w}}$ is an indicator of the thermodynamic stability of hydrates, with lower values implying greater tendency for hydration. Differences in the critical water activities of drug and cocrystal hydrates may be due to differences in the crystal structure, hydrogen bond interactions between water, drug, and/or coformer, as well as differences in solubility and free energy of these materials.

\section{CONCLUSIONS}

Coformers, excipients, and cosolvents that modulate the water activity of solutions can affect cocrystal hydrate/anhydrous stability and induce transformation to or from anhydrous cocrystal. This is shown for THP-CTA and CBZ-4-ABA cocrystal hydrates and anhydrous forms. Cocrystal stability is determined by both water and coformer activities and can be evaluated by suspension equilibration studies by measurement of both cocrystal component concentrations at equilibrium. Key parameters to identify anhydrous and hydrated cocrystal stability domains are eutectic concentrations and critical water activity. Vapor phase equilibration studies should take into account the purity of cocrystal phases since the presence of water-soluble coformers as trace level impurities are expected to alter the observed hygroscopic behavior of cocrystals.

\section{ACKNOWLEDGMENTS}

We are grateful to Dr. Sreenivas Reddy for fruitful discussions and assistance with cocrystal stability studies. We gratefully acknowledge financial support from the Purdue-Michigan consortium for the Study of Supramolecular Assemblies and Solid-state Properties, the Fred W. Lyons and Warner Lambert/Parke Davis Fellowships, College of Pharmacy, University of Michigan, Ann Arbor, MI.

\section{REFERENCES}

1. Good DJ, Rodríguez-Hornedo N. 2009. Solubility advantage of pharmaceutical cocrystals. Cryst Growth Des 9:2252-2264.
2. Aakeroy C, Forbes S, Desper J. 2009. Using cocrystals to systematically modulate aqueous solubility and melting behavior of an anticancer drug. J Am Chem Soc 131:17048.

3. Byrn SR, Pfeiffer RR, Stowell JG. 1999. Solid-state chemistry of drugs, 2nd edition. West Lafayette, IN: SSCI, Inc.

4. Bethune SJ, Huang N, Jayasankar A, Rodríguez-Hornedo N. 2009. Understanding and predicting the effect of cocrystal components and $\mathrm{pH}$ on cocrystal solubility. Cryst Growth Des 9:3976-3988.

5. Bak A, Gore A, Yanez E, Stanton M, Tufekcic S, Syed R. 2008. The cocrystal approach to improve the exposure of a waterinsoluble compound: AMG 517 sorbic acid cocrystal characterization and pharmacokinetics. J Pharm Sci 97:3942-3956.

6. Schultheiss N, Newman A. 2009. Pharmaceutical cocrystals and their physicochemical properties. Cryst Growth Des 9: 2950-2967.

7. McNamara DP, Childs SL, Giordano J, Iarriccio A, Cassidy J, Shet MS, Mannion R, O’Donnell E, Park A. 2006. Use of a glutaric acid cocrystal to improve oral bioavailability of a low solubility API. Pharm Res 23:1888-1897.

8. Trask AV, Motherwell WDS, Jones W. 2005. Pharmaceutical cocrystallization: Engineering a remedy for caffeine hydration. Cryst Growth Des 5:1013-1021.

9. Rager T, Hilfiker R. 2009. Stability domains of multi-component crystals in ternary phase diagrams. Z Phys Chem 223:793-813.

10. Jayasankar A, Reddy LS, Bethune SJ, Rodríguez-Hornedo N. 2009. The role of cocrystal and solution chemistry on the formation and stability of cocrystals with different stoichiometry. Cryst Growth Des 9:889-897.

11. Trask AV, Motherwell WDS, Jones W. 2006. Physical stability enhancement of theophylline via cocrystallization. Int J Pharm 320:114-123.

12. Karki S, Friščić T, Jones W, Motherwell WDS. 2007. Screening for pharmaceutical cocrystal hydrates via neat and liquidassisted grinding. Mol Pharm 4:347-354.

13. Zhu H, Yuen C, Grant DJW. 1996. Influence of water activity in organic solvent+water mixtures on the nature of the crystallizing drug phase. 1. Theophylline. Int J Pharm 135:151160.

14. Zhu H, Grant DJW. 1996. Influence of water activity in organic solvent plus water mixtures on the nature of the crystallizing drug phase2. Ampicillin. Int J Pharm 139:33-43.

15. Morris KR. 1999. Structural aspects of hydrates and solvates. In: Britain HG, editor. Polymorphism in pharmaceutical solids, 1st edition. New York: Marcel Dekker, Inc. pp 126-180.

16. Stephenson G, Stowell J, Toma P, Pfeiffer R, Byrn S. 1997. Solid-state investigations of erythromycin A dihydrate: Structure, NMR spectroscopy, and hygroscopicity. J Pharm Sci 86:1239-1244.

17. Salameh AK, Taylor LS. 2006. Physical stability of crystal hydrates and their anhydrates in the presence of excipients. J Pharm Sci 95:446-461.

18. Rodríguez-Hornedo N, Lechuga-Ballesteros D, Hsiu-Jean W. 1992. Phase transition and heterogeneous/epitaxial nucleation of hydrated and anhydrous theophylline crystals. Int J Pharm 85:149-162

19. Wanchai C, Byrn SR, Narueporn S. 2008. Solid state interconversion between anhydrous norfloxacin and its hydrates. J Pharm Sci 97:473-489.

20. Li Y, Chow PS, Tan RBH, Black SN. 2008. Effect of water activity on the transformation between hydrate and anhydrate of carbamazepine. Org Process Res Dev 12:264-270.

21. Qu H, Louhi-Kultanen M, Kallas J. 2006. Solubility and stability of anhydrate/hydrate in solvent mixtures. Int J Pharm 321:101-107.

22. Ticehurst MD, Storey RA, Watt C. 2002. Application of slurry bridging experiments at controlled water activities to predict 
the solid-state conversion between anhydrous and hydrated forms using theophylline as a model drug. Int J Pharm 247: $1-10$.

23. Khankari RK, Grant DJW. 1995. Pharmaceutical hydrates. Thermochim Acta 248:61-79.

24. Childs SL, Rodríguez-Hornedo N, Reddy LS, Jayasankar A, Maheshwari C, McCausland L, Shipplett R, Stahly BC. 2008. Screening strategies based on solubility and solution composition generate pharmaceutically acceptable cocrystals of carbamazepine. Cryst Eng Commun 11:856-864.

25. Friscic T, Fabian L, Burley JC, Reid DG, Duer MJ, Jones W. 2008. Exploring the relationship between cocrystal stability and symmetry: Is Wallach's rule applicable to multi-component solids? Chem Commun 14:1644-1646.

26. Chiarella RA, Davey RJ, Peterson ML. 2007. Making cocrystals-The utility of ternary phase diagrams. Cryst Growth Des $7: 1223-1226$.

27. Rodríguez-Hornedo N, Nehm SJ, Seefeldt KF, Pagán-Torres Y, Falkiewicz CJ. 2006. Reaction crystallization of pharmaceutical molecular complexes. Mol Pharm 3:362-367.

28. Zhang GGZ, Henry RF, Borchardt TB, Lou X. 2007. Efficient cocrystal screening using solution-mediated phase transformation. J Pharm Sci 96:990-995.

29. Fleischman SG, Kuduva SS, McMahon JA, Moulton B, Walsh RDB, Rodríguez-Hornedo N, Zaworotko MJ. 2003. Crystal engineering of the composition of pharmaceutical phases: Multiple-component crystalline solids involving carbamazepine. Cryst Growth Des 3:909-919.

30. Bucar D, Henry R, Lou X, Duerst R, MacGillivray L, Zhang G. 2009. Cocrystals of caffeine and hydroxybenzoic acids composed of multiple supramolecular heterosynthons: screening via solution-mediated phase transformation and structural characterization. Cryst Growth Des 9:1932-1943.

31. Rodríguez-Hornedo N, Nehm SJ, Jayasankar A. 2006. Cocrystals: design, properties and formation mechanisms. In: Swarbrick J, editor. Encyclopedia of pharmaceutical technology, 3rd edition. New York: Informa Health Care. pp 615-635.

32. McMahon JA, Bis JA, Vishweshwar P, Shattock TR, McLaughlin OL, Zaworotko MJ. 2005. Crystal engineering of the composition of pharmaceutical phases. 3. Primary amide supramolecular heterosynthons and their role in the design of pharmaceutical cocrystals. Z Kristallogr 220:340-350.

33. Apelblat A, Dov M, Wisniak J, Zabicky J. 1995. The vapor pressure of water over saturated aqueous solutions of malic, tartaric, and citric acids, at temperature from $288 \mathrm{~K}$ to $323 \mathrm{~K}$. J Chem Thermodyn 27:35-41.

34. Levien BJ. 1955. A physicochemical study of aqueous citric acid solutions. J Phys Chem 59:640-644.

35. Salameh AK, Mauer LJ, Taylor LS. 2006. Deliquescence lowering in food ingredient mixtures. J Food Sci 71:E10-E16.

36. Gracin S, Rasmuson AC. 2004. Polymorphism and crystallization of p-aminobenzoic acid. Cryst Growth Des 4:1013-1023.
37. Nehm SJ, Rodríguez-Spong B, Rodríguez-Hornedo N. 2006. Phase solubility diagrams of cocrystals are explained by solubility product and solution complexation. Cryst Growth Des 6:592-600.

38. Bell G, Janssen AEM, Halling PJ. 1997. Water activity fails to predict critical hydration level for enzyme activity in polar organic solvents: Interconversion of water concentrations and activities. Enzyme Microb Technol 20:471-477.

39. O'Brien FEM. 1948. The control of humidity by saturated salt solutions. J Sci Instrum 25:73-76.

40. Apelblat A. 1992. The vapour pressures of water over saturated aqueous solutions of barium chloride, magnesium nitrate, calcium nitrate, potassion carbonate, and zinc sulfate at temperatures from $283 \mathrm{~K}$ to $313 \mathrm{~K}$. J Chem Thermodyn 24:619626.

41. Jayasankar A, Good DJ, Rodríguez-Hornedo N. 2007. Mechanisms by which moisture generates cocrystals. Mol Pharm 4:360-372.

42. Good DJ, Rodríguez-Hornedo N. 2010. Cocrystal eutectic constants and prediction of solubility behavior. Cryst Growth Des 10:1028-1032.

43. Apelblat A, Dov M, Wisniak J, Zabicky J. 1995. Osmotic and activity coefficients of $\mathrm{HO}_{2} \mathrm{CCH}_{2} \mathrm{C}(\mathrm{OH})\left(\mathrm{CO}_{2} \mathrm{H}\right) \mathrm{CH}_{2} \mathrm{CO}_{2} \mathrm{H}$ (citric acid) in concentrated aqueous solutions at temperatures from $298.15 \mathrm{~K}$ to $318.15 \mathrm{~K}$. J Chem Thermodyn 27:347-353.

44. Maffia MC, Meirelles AJA. 2001. Water activity and $\mathrm{pH}$ in aqueous polycarboxylic acid systems. J Chem Eng Data 46:582587.

45. Peng C, Chow AHL, Chan CK. 2001. Hygroscopic study of glucose, citric acid, and sorbitol using an electrodynamic balance: Comparison with unifac predictions. Aerosol SciTechnol 35:753-758.

46. Salameh AK, Taylor LS. 2006. Deliquescence-induced caking in binary powder blends. Pharm Dev Technol 11:453-464.

47. Otsuka M, Kaneniwa N, Kawakami K, Umezawa O. 1990. Effect of surface characteristics of theophylline anhydrate powder on hygroscopic stability. J Pharm Pharmacol 42:606610.

48. Clegg S, Seinfeld J. 2006. Thermodynamic models of aqueous solutions containing inorganic electrolytes and dicarboxylic acids at $298.15 \mathrm{~K} .1$. The acids as nondissociating components. J Phys Chem A 110:5692-5717.

49. Kontny MJ, Conners JJ. 2002. Water sorption of drugs and dosage forms. In: Swarbrick J, Boylan JC, editors. Encyclopedia of pharmaceutical technology, 2nd edition. New York: Marcel Dekker, Inc. pp 2970-2986.

50. Debnath S, Suryanarayanan R. 2004. Influence of processinginduced phase transformations on the dissolution of theophylline tablets. AAPS PharmSciTech 5:1-11.

51. Peng C, Chan MN, Chan CK. 2001. The hygroscopic properties of dicarboxylic and multifunctional acids: measurements and UNIFAC predictions. Environ Sci Technol 35:4495-4501. 